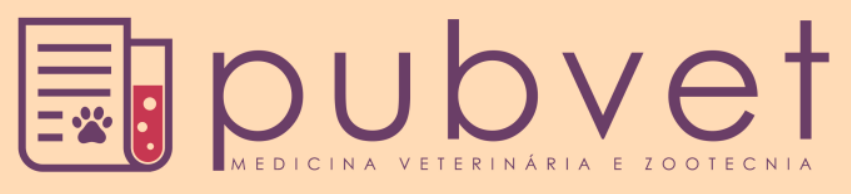

https://doi.org/10.22256/pubvet.v12n3a48.1-13

\title{
Aplicabilidade da proteína haptoglobina como bioindicador de saúde na bovinocultura de corte e leite
}

\author{
Bruno Lopes Bastos ${ }^{\ominus 1 *}$, José Tadeu Raynal Rocha-Filho ${ }^{\bullet}$, Ítalo Paulino Santana ${ }^{\ominus}$, \\ Roberto Meyer ${ }^{\natural}$, José Eugênio Guimarães ${ }^{\bullet} 5$ \\ ${ }^{I}$ Professor da Universidade Federal da Bahia, Instituto Multidisciplinar em Saúde - Campus Anísio Teixeira, Vitória da Conquista - BA, Brasil. \\ ${ }^{2}$ Doutor em Biotecnologia, Universidade Federal da Bahia, Instituto de Ciências da Saúde, Salvador-BA, Brasil. E-mail: jtraynal@ hotmail.com \\ ${ }^{3}$ Graduando do curso de Biotecnologia da Universidade Federal da Bahia, Instituto Multidisciplinar em Saúde - Campus Anísio Teixeira, \\ Vitória da Conquista - BA, Brasil.E-mail: italopaulinos@hotmail.com \\ ${ }^{4}$ Professor da Universidade Federal da Bahia, Instituto de Ciências da Saúde, Salvador-BA, Brasil. E-mail: meyer.roberto@gmail.com \\ ${ }^{5}$ Professor da Universidade Federal da Bahia, Escola de Medicina Veterinária e Zootecnia, Salvador-BA, Brasil. E-mail: jeugenio28@ globo.com \\ *Autor para correspondência, E-mail: blbastos@ufba.br.
}

RESUMO. A haptoglobina é a principal proteína de fase aguda em ruminantes, sendo o seu uso amplamente pesquisado na clínica médica animal como biomarcador precoce de processos inflamatórios, infecciosos, danos teciduais e condições de estresse. Isto se deve ao fato de seus valores sanguíneos se apresentarem próximos de $0,05 \mathrm{mg} / \mathrm{ml}$ em bovinos saudáveis, e se elevarem em mais de 100 vezes durante a ocorrência das condições patológicas, antes mesmo que surjam sinais clínicos e ocorram alterações no leucograma. Com estas características, a haptoglobina apresenta um alto potencial para uso em diferentes segmentos da cadeia produtiva pecuária brasileira, sendo o objetivo desta revisão de literatura compilar os trabalhos científicos que utilizaram a esta proteína como bioindicador de saúde animal na bovinocultura de corte e leite. Verificou-se que, na pecuária de corte, a quantificação da haptoglobina pode contribuir com a triagem dos animais na inspeção ante morten, permitindo a identificação dos animais com doença subclínica que não foram diagnosticados pela inspeção visual; pode também contribuir para a verificação do bem-estar animal nos momentos após o transporte e pré-abate, evitandose o abate de animais com possíveis quadros de estresse que possam alterar a qualidade dos produtos cárneos. Na pecuária leiteira, a haptoglobina pode contribuir diretamente com a identificação de vacas com diferentes níveis de mastite clínica e subclínica, além de permitir a identificação de lotes de leite com alta concentração de células somáticas ou oriundos de rebanhos acometidos por agentes infecciosos. Para que seja fomentada a sua inclusão como ferramenta de monitoramento dentro cadeia produtiva pecuária brasileira, para uso por ambos médicos veterinários e estabelecimentos industriais de produtos de origem animal, recomenda-se a realização de estudos de validação do uso da haptoglobina como bioindicador de saúde animal em parceria com empresas do setor, de forma que a sua utilidade prática seja verificada diretamente pelos profissionais envolvidos.

Palavras chave: diagnóstico, pecuária, proteína de fase aguda, sanidade animal

\section{Applicability of haptoglobin as a health bioindicator in beef and dairy cattle}

ABSTRACT. Haptoglobin is the main acute phase protein in ruminants, and its use is being widely investigated in animal clinics as an early biomarker of inflammation, infection, tissue damage and stress conditions. This occurs due to the fact that this protein presents blood values close to $0.05 \mathrm{mg} / \mathrm{ml}$ in healthy cattle, but it increases more than 100 times during the pathological conditions, even before clinical signs appear and changes in 
the leukogram occur. Considering these characteristics, haptoglobin has a high potential for use in different areas of the Brazilian livestock industry. Thus, the aim of this literature review is to compile the scientific studies that used this protein as a bioindicator of animal health in beef and dairy cattle. In beef cattle, it was verified that the quantification of haptoglobin may contribute to the screening of animals in the ante morten inspection, allowing the identification of animals with subclinical disease that were not diagnosed by visual inspection; can also contribute to the verification of animal welfare in the moments after transportation and pre-slaughter, avoiding the slaughter of animals with possible stresses that may alter the quality of the meat products. In dairy cattle, haptoglobin can directly contribute to the identification of cows with different levels of clinical and subclinical mastitis, besides allowing the identification of lots of milk with high concentration of somatic cells or from herds affected by infectious agents. In order to promote its inclusion as a monitoring tool within the Brazilian livestock industry, for use by both veterinarians and industrial establishments of animal products, it is recommended to carry out validation studies on the use of haptoglobin as an animal health bioindicator in partnership with companies of the sector, so that its practical utility is verified directly by the professionals involved.

Keywords: diagnosis, livestock, acute phase protein, animal health

\section{Aplicabilidad de la proteína haptoglobina como bioindicador de salud en la ganadería de carne y leche}

RESUMEN. La haptoglobina es la principal proteína de fase aguda en rumiantes, siendo su uso ampliamente investigado en la clínica médica animal como biomarcador precoz de procesos inflamatorios, infecciosos, daños en tejido y condiciones de estrés. Esto se debe al hecho de que sus valores sanguíneos se presentan cerca de $0,05 \mathrm{mg} / \mathrm{ml}$ en bovinos sanos, y se elevan en más de 100 veces durante la presencia de condiciones patológicas, incluso antes que aparezcan signos clínicos y ocurran alteraciones en el leucograma. Con estas características, la haptoglobina presenta un alto potencial para uso en diferentes segmentos de la cadena productiva pecuaria brasileña, siendo el objetivo de esta revisión de literatura compilar los trabajos científicos que utilizaron esta proteína como bioindicador de salud animal en la ganadería de carne y leche. Se verificó que, en la ganadería de carne, la cuantificación de la haptoglobina puede contribuir con la clasificación de los animales en la inspección ante morten, permitiendo la identificación de los animales con enfermedad subclínica que no fueron diagnosticados por la inspección visual; también puede contribuir con la verificación del bienestar animal en los momentos posteriores al transporte y presacrificio, evitando el sacrificio de animales con posibles cuadros de estrés que puedan alterar la calidad de los productos cárnicos. En la ganadería lechera, la haptoglobina puede contribuir directamente con la identificación de vacas con diferentes niveles de mastitis clínica y subclínica, además de permitir la identificación de lotes de leche con alta concentración de células somáticas o provenientes de rebaños afectados por agentes infecciosos. Para que sea fomentada su inclusión como herramienta de monitoreo dentro cadena productiva pecuaria brasileña, para uso por ambos médicos veterinarios y establecimientos industriales de productos de origen animal, se recomienda la realización de estudios de validación del uso de la haptoglobina como bioindicador de salud animal en asociación con empresas del sector, de forma que su utilidad práctica sea verificada directamente por los profesionales involucrados.

Palabras clave: diagnóstico, pecuaria, proteína de fase aguda, sanidad animal

\section{Introdução}

A haptoglobina, por ser uma proteína de fase aguda, tem sido utilizada na clínica médica humana e veterinária como biomarcador precoce de processos inflamatórios e infecciosos (Tothova et al., 2014); isto se deve ao fato de seus níveis plasmáticos elevarem-se significantemente nas primeiras horas após o início da patologia, antes mesmo que surjam sinais clínicos e ocorram 
alterações no leucograma (Ceciliani et al., 2012; Carvalho et al., 2012). A utilidade de um marcador deste tipo transcende os limites da clínica, podendo ser aplicada no monitoramento sanitário de animais de abate, o qual deve ser realizado por médicos veterinários do serviço oficial de inspeção sanitária de cada país (Ndou et al., 2011; Klauke et al., 2013). Alguns estabelecimentos da União Européia já estão em processo de implementação de sistemas de quantificação da haptoglobina de suínos e bovinos durante o abate, objetivando identificar animais portadores de infecções que representam risco à segurança alimentar e saúde pública, de forma a orientar as ações executadas na inspeção post-mortem (Blagojevic et al., 2011; Soler et al., 2013). A análise laboratorial pode ser conduzida por meio de ensaios imunológicos ou bioquímicos, os quais já estão disponíveis comercialmente (Cooke \& Arthington 2012; Segawa et al., 2013; Czopowicz et al., 2017), e como material biológico podem ser utilizadas amostras de sangue, saliva e urina, assim como amostras de carne, que após trituradas liberam seus fluidos incorporados (Soler et al., $\underline{2013}$ ).

Além da aplicabilidade na cadeia produtiva da carne, diferentes estudos tem demonstrado que esta proteína também permite a identificação de vacas portadoras de mastite subclínica utilizando o próprio leite como amostra biológica (Grönlund et al., 2003). Tais evidências motivaram o desenvolvimento de sistemas de biosensores de quantificação de haptoglobina para uso em laticínios, com o intuito de monitorar a qualidade do leite e seus produtos derivados (Akerstedt et al., 2006; Akerstedt et al., 2008). Outras potenciais aplicabilidades da haptoglobina que podem ser verificadas na literatura especializada envolvem a avaliação de condições de estresse e do bem-estar animal (Gómez-Laguna et al., 2011).

No Brasil, embora a quantificação da haptoglobina seja utilizada primordialmente em pesquisas científicas de caráter clínico com diferentes espécies animais (Battisti et al., 2013; Filippo et al., 2014), a sua inclusão como ferramenta dentro da cadeia produtiva pecuária bovina pode contribuir estrategicamente para aprimorar a qualidade dos produtos cárneos e lácteos brasileiros perante o mercado externo e interno. Diante da sua potencial utilidade para o setor produtivo, esta revisão de literatura tem como objetivo compilar os trabalhos científicos que utilizaram a haptoglobina como bioindicador de saúde animal na bovinocultura de corte e leite, de forma a fomentar a sua inclusão como ferramenta de monitoramento dentro cadeia produtiva pecuária brasileira.

A palavra introdução deve estar em negrito e sem recuo. A introdução não deve exceder 2.000 caracteres (caracteres com espaço) e justifica brevemente a pesquisa, especifica a hipótese a ser testada e os objetivos. Uma extensa discussão da literatura relevante deve ser incluída na discussão.

\section{Resposta de fase aguda e haptoglobina}

A resposta de fase aguda de um organismo constitui uma reação sistêmica desencadeada por distúrbios na homeostasia causados por processos inflamatórios, infecciosos, traumáticos e neoplásicos. Um dos sinais desta reação é o incremento da secreção hepática de proteínas de fase aguda na circulação sanguínea, que ocorre antes mesmo das alterações no leucograma e expressão de sinais clínicos (Ceciliani et al., 2012). Uma das proteínas mais amplamente estudadas na medicina humana e veterinária é a haptoglobina (Eckersall et al., 2007), a qual desempenha importante papel fisiológico e imuno-regulador em processos infecciosos e inflamatórios (Arredouani et al., 2005).

Bioquimicamente, a haptoglobina é uma glicoproteína que faz parte do grupo das alfa-2globulinas e difere em sua forma entre as espécies de mamíferos (Elson 1974). A sua função primária é prevenir a perda de ferro sanguíneo pela formação de complexos estáveis com a hemoglobina livre no sangue (Laurell \& Nyman, 1957). Por este motivo, esta proteína desempenha uma atividade bacteriostática através da restrição do ferro necessário ao crescimento bacteriano (Eaton et al., 1982). Outras funções já foram atribuídas à haptoglobina, incluindo ações imunoreguladoras relacionadas à quimiotaxia leucocitária, capacidade de fagocitose e atividade bactericida dos granulócitos (Rossbacher et al., 1999), assim como sobre a linfoproliferação, promovendo a atenuação da resposta imune $\mathrm{Th} 2 \mathrm{e}$ a predominância da resposta imune Th1 (Arredouani et al., 2003; Arredouani et al., 2005).

Durante processos infecciosos e inflamatórios, uma larga produção hepática de haptoglobina ocorre a partir de estímulos gerados através da secreção das citocinas pró-inflamatórias como o fator de necrose tumoral alfa (TNF-alfa) e das interleucinas 1 e 6 (IL-1 e IL-6) pelos macrófagos no local da lesão (Kurash et al., 2004; Murata et al., 2004), conforme ilustrado por peio da Figura 
1. Além da produção hepática, leucócitos migram para o sítio inicial da infecção e contribuem para a expressão desta proteína in loco, sendo também sintetizada e liberada em menor quantidade por neutrófilos em resposta à sua ativação (Thielen et al., 2005; Theilgaard-Mönch et al., 2006). Dessa forma, a haptoglobina tem suas concentrações séricas elevadas drasticamente em resposta aos processos patológicos. A exemplo, bovinos saudáveis apresentam níveis médios de haptoglobina sanguínea de $0,01 \mathrm{~g} / \mathrm{L}$; enquanto animais com quadros infecciosos agudos podem apresentar níveis médios de até 1,62 g/L (Skinner et al., 1991; Eckersall et al., 1999). Diversos pesquisadores comprovaram a utilidade clínica da haptoglobina para o monitoramento da ocorrência e gravidade de reações inflamatórias em bovinos com mastite, pneumonia, enterite, peritonite, endocardite, abcessos, endometrite e outras condições infecciosas naturais ou experimentais (Ceciliani et al., 2012).

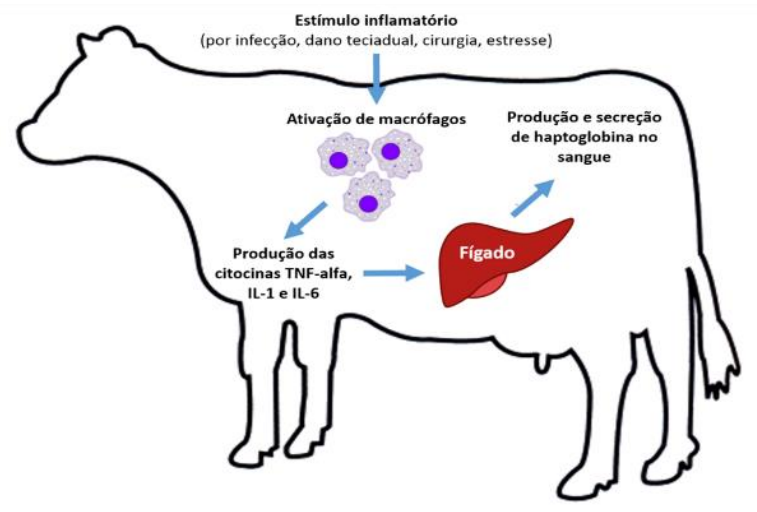

Figura 1. Resposta de fase aguda em bovinos. A ocorrência de estímulos inflamatórios de diferentes origens induz a ativação local de mecanismos celulares de defesa do animal, principalmente macrófagos, que por sua vez produzem as citocinas pró-inflamatórias, fator de necrose tumoral alfa (TNF-alfa), interleucina 1 (IL-1) e interleucina 6 (IL-6). Estas ativam a produção hepática e secreção da haptoglobina no sangue.

Diante do potencial como biomarcador de infecções e inflamações, além de outras condições patológicas, a haptoglobina se tornou foco de inúmeras investigações sobre a sua aplicabilidade no monitoramento e diagnóstico de doenças de rebanhos animais de produção, principalmente aquelas com caráter sub-clínico, sem expressão nítida de sintomas (Petersen et al., 2004). Justamente a possibilidade de identificação de animais com doença sub-clínica despertou o interesse no uso da haptoglobina como bioindicador de saúde e estresse animal na inspeção ante-mortem em frigoríficos (Tothova et al., 2014).

\section{Uso da haptoglobina como bioindicador sanitário na pecuária de corte}

Diversos países têm revisado os procedimentos recomendados para as ações envolvidas na inspeção sanitária de produtos de origem animal; conforme pesquisas, o sistema tradicional de inspeção, baseado principalmente em avaliações visuais, não é capaz de identificar parte dos riscos à saúde públicos originados por contaminação microbiana de produtos nas fases de seu processamento. Essa modernização do sistema de inspeção tem objetivado melhorias na segurança alimentar por meio de aprimoramentos do padrão sanitário dos animais de produção (Hill et al., 2014). Neste contexto, a haptoglobina tem sido sugerida como um biomarcador multiuso para diferentes setores da cadeia produtiva de produtos de origem animal, com grandes perspectivas de aplicação em frigoríficos (Ndou et al., 2011).

$\mathrm{Na}$ cadeia produtiva dos produtos cárneos, a haptoglobina apresenta-se como um promissor biomarcador para monitorar a saúde de animais de abate. Animais saudáveis apresentam níveis desta proteína praticamente indetectáveis, já animais portadores de doenças sempre apresentam concentrações sanguíneas significantemente elevadas de haptoglobina (Gruys et al., 2005). Diante deste potencial, pesquisadores sugerem o seu uso como exame padrão pré-abate para a triagem e separação de animais com doença subclínica em frigoríficos, principalmente aqueles que representam risco à saúde pública (Saini \& Webert, 1991; Saini et al., 1998). Para a validação da haptoglobina como ferramenta auxiliar na inspeção sanitária ante-mortem de bovinos, trabalhos foram realizados junto ao serviço veterinário oficial de diferentes países, sendo os resultados muito promissores.

Na Finlândia, realizou-se um trabalho para verificar o valor da haptoglobina como ferramenta auxiliar na inspeção sanitária ante-mortem de bovinos, sendo avaliado um grupo de 80 vacas abatidas emergencialmente; os valores da haptoglobina quantificada durante a inspeção ante-mortem foram confrontados com os achados patológicos registrados na inspeção post-mortem. Animais com lesões mínimas ou sem patologias detectadas apresentaram o valor médio de $0,01 \pm$ $0,01 \mathrm{~g} / \mathrm{L}$, enquanto que todos os animais portadores de patologias registradas pelos veterinários do serviço de inspeção oficial apresentaram valores médios acima de $0,60 \mathrm{~g} / \mathrm{L}$. As altas concentrações de haptoglobina sérica 
estavam associadas a quadros de traumas musculares $(0,66 \pm 0,13 \mathrm{~g} / \mathrm{L})$, mastites $(0,67 \pm$ $0,16 \mathrm{~g} / \mathrm{L})$, artrites $(0,79 \pm 0,23 \mathrm{~g} / \mathrm{L})$, infecções generalizadas $(0,69 \pm 0,18 \mathrm{~g} / \mathrm{L})$ e quadros miscelâneos $(0,67 \pm 0,12 \mathrm{~g} / \mathrm{L})$. Estatisticamente, a diferença entre os valores de animais saudáveis e doentes foi altamente significante (p-0,001), permitindo-se concluir que a haptoglobina constitui um bioindicador de altíssima sensibilidade para a identificação de bovinos doentes. Deve ser destacado que os grupos de vacas com artrite e infecções generalizadas apresentaram $62,1 \%$ e $100 \%$ de carnes condenadas pela inspeção, respectivamente (Hirvonen, 1997).

Um grupo de pesquisadores do Reino Unido realizou um trabalho semelhante com animais de abate, demonstrando que a haptoglobina apresenta uma especificidade de $76 \%$ para a diferenciação de enfermidades agudas e crônicas, visto que os animais portadores de condições patológicas agudas apresentaram valores médios desta proteína próximos a $0,81 \mathrm{~g} / \mathrm{L}$. Dentre os quadros agudos registrados pelo serviço de inspeção antemortem, destacam-se pneumonias, peritonites, pericardites, pleurisias, nefrites, artrites, mastites e fraturas ósseas. Conforme os resultados destas pesquisas, o estabelecimento de um ponto de corte de $0,18 \mathrm{~g} / \mathrm{L}$ permitiria a identificação de bovinos suspeitos de portarem alguma das patologias citadas ainda na avaliação ante-mortem; esta triagem direcionaria e otimizaria as ações de inspeção post-mortem, de forma a aprimorar a segurança alimentar dos produtos cárneos (Horadagoda et al., 1999; Tourlomoussis et al., 2004). Trabalhos mais recentes demonstraram o poder preditivo da haptoglobina em identificar bovinos portadores de broncopneumonias, enterites, infestações parasitárias hepáticas (Fasciola sp. e Echinococcus sp.) e metrites, além de outras patologias já citadas anteriormente (Blagojevic et al., 2011). É válido destacar que o resultado promissor obtido com o abate de bovinos tem estimulado o uso da haptoglobina como biomarcador em outras cadeias produtivas de carne; uma pesquisa já foi realizada em um frigorífico com ovinos, no qual a mensuração desta proteína no soro permitiu a identificação dos animais com doenças subclinicas ainda na inspeção ante-mortem. Também foi constatada uma estrita correlação entre os níveis da proteína e a presença e severidade de patologias detectadas em órgãos internos na inspeção post-mortem (Kostro et al., 2009).
Além de ser útil para a triagem sanitária dos animais de abate, a haptoglobina também tem sido utilizada para complementar o monitoramento de animais portadores de doenças infecciosas de notificação obrigatória. Infecções naturais e experimentais de bovinos com o vírus da Febre Aftosa do sorotipo $\mathrm{O}$ indicaram que a elevação da haptoglobina sérica está associada com o momento da viremia e aparecimento de sinais clínicos, sendo sugerido o uso desta proteína como biomarcador de infecção aguda pelo vírus em questão (Höfner et al., 1994; Stenfeldt et al., 2011). Outros estudos também apontam o potencial da haptoglobina para monitoramento de Brucelose (Sharifiyazdia et al., 2012) e Encefalopatias Espongiformes Transmissíveis (Meling et al., 2012).

\section{Uso da haptoglobina como bioindicador de mastite e qualidade do leite}

A inflamação dos quartos mamários, conhecida como mastite, permanece como uma das maiores preocupações encontradas na exploração de animais de leite e é considerada a doença mais importante do rebanho leiteiro sob o ponto de vista econômico; a colonização do tecido secretor do úbere por agentes patogênicos e a consequente resposta inflamatória acarretam na menor quantidade de leite produzido (Carneiro et al., 2009). Além disso, os microrganismos contaminantes reduzem a qualidade do leite dos quartos mamários afetados e proporcionam alterações na sua composição, que resultam em interferência na tecnologia industrial para elaboração de derivados. Vale ressaltar que a mastite também apresenta aspectos de saúde pública, considerando-se a veiculação de patógenos causadores de doença em humanos através do leite (Bandeira et al., 2013).

Atualmente, o teste considerado "padrão ouro" para monitoramento da saúde do úbere e da qualidade do leite em empreendimento leiteiros é a Contagem de Células Somáticas (CCS/mL de leite), conforme a Instrução Normativa $\mathrm{N}^{\circ} 62$ do Ministério da Agricultura, Pecuária e Abastecimento - MAPA (Langoni, 2013). Embora utilizado amplamente por laticínios no mundo, pesquisas demonstraram que o teste apresenta uma baixa sensibilidade para a detecção de mastites sub-clínicas, com valores entre $56 \%$ e 76\% (Sargeant et al., 2001; Middleton et al., 2004). Pesquisadores do setor sugeriram a produção de novas tecnologias para monitoramento de mastites e qualidade do leite 
que possam ser adaptadas ao uso em ambientes industriais de recepção e beneficiamento leiteiro. Neste sentido, a haptoglobina foi considerada um promissor candidato para o monitoramento de

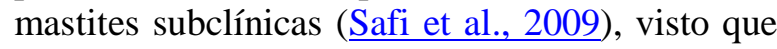
durante a inflamação do úbere ocorre uma resposta de fase aguda clássica, com alta produção hepática de haptoglobina e sua secreção na circulação sanguínea (Vels et al., 2009).

Os resultados de um estudo utilizando a haptoglobina como biomarcador de mastite bovina foram publicados já em 1996, quando demonstrou-se que vacas apresentaram elevação significante dos níveis sanguíneos desta proteína a partir de 12 horas após a infecção experimental dos quartos mamários por Escherichia coli. Após 72 horas, a haptoglobina já apresentava um incremento de 52 vezes em relação ao seu valor basal (Salonen et al., 1996). Trabalhos posteriores também demonstraram que, além de ser bioindicador da ocorrência de mastite, a concentração sanguínea de haptoglobina apresentava uma forte associação com os sinais clínicos da enfermidade, correlacionando-se positivamente com a severidade da doença (mastite sub-clínica, clínica aguda e hiper-aguda) (Hirvonen et al., 1999; Ohtsuka et al., 2001; Hisaeda et al., 2011).

Para que a haptoglobina pudesse ser aplicada em monitoramentos de larga escala em laticínios, foram iniciados trabalhos de padronização da detecção desta proteína diretamente em amostras de leite. Demonstrou-se que a mensuração da haptoglobina sanguínea para o diagnóstico de vacas com mastite clínica apresenta sensibilidade e especificidade de $82 \%$ e $94 \%$, respectivamente, enquanto a sua quantificação diretamente no leite apresenta níveis de sensibilidade e especificidade de $86 \%$ e $100 \%$, respectivamente (Eckersall et al., 2001), além de permitir a distinção de animais com mastite aguda e mastite crônica (Grönlund et al., 2003). As comprovações da participação fisiológica direta da haptoglobina na resposta imune inata do úbere ocorreram apenas posteriormente. Constatou-se experimentalmente que durante a ocorrência de mastite sub-clínica causada por Staphylococcus aureus, a elevação da Haptoglobina no leite em níveis significantes precedia a elevação da proteína no sangue (Eckersall et al., 2006); através de testes de quantificação de RNAm, foi descoberto que ocorria expressão de haptoglobina diretamente por células do epitélio mamário e neutrófilos infiltrados durante o quadro de mastite, sendo esta a principal fonte desta proteína no leite (Hiss et al., 2004; Thielen et al., 2007; Lai et al., 2009).

A partir destas comprovações, a haptoglobina passou a ser considerada definitivamente como um bioindicador de mastite, que apresenta elevação em suas concentrações no leite na ausência de alterações macroscópicas e precede o aparecimento dos sinais clínicos. Conforme dados experimentais, a dosagem de haptoglobina no leite apresenta uma maior acurácia que a sua análise no sangue para o diagnóstico de mastite subclínica, sendo sugerida a incorporação deste teste como alternativa ao uso da contagem de células somáticas nos laticínios (Safi et al., 2009). Outras pesquisas também comprovaram o potencial da haptoglobina mensurada em amostras de leite como biomarcador da ocorrências de mastites clínicas e sub-clínicas, registrando resultados semelhantes (Suojala et al., 2008; Wenz et al., 2010; Pyörälä et al., 2011), conforme ilustrado na Figura 2.

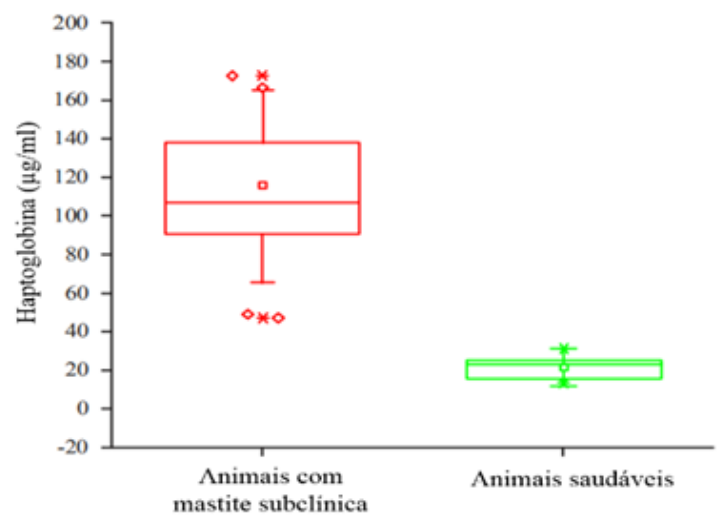

Figura 2. Box plots demonstrando a distribuição dos valores de haptoglobina quantificada diretamente de amostras de leite para detecção de animais com mastite subclínica. Adaptado de Singh et al. (2015).

Além de ser biomarcador de mastite clínica e subclínica, a haptoglobina constitui um bioindicador da qualidade do leite. Diversos pesquisadores verificaram uma forte correlação positiva entre as concentrações de haptoglobina no leite e os respectivos valores de contagem de células somáticas (CCS) (Nielsen et al., 2004; Grönlund et al., 2005; Akerstedt et al., 2007) foi descoberto também que altos valores de haptoglobina estão associados a um menor teor de caseína e lactose no produto, o que potencialmente prejudica o beneficiamento do leite e a produção de derivados (Akerstedt et al., 2009). Outra pesquisa recente demonstrou o potencial da haptoglobina como bioindicador de contaminação do leite, sendo demonstrada experimentalmente a 
forte associação entre os seus níveis e a quantidade de DNA microbiano detectado na amostra (Kalmus et al., 2013).

Diante do potencial da haptoglobina como biomarcador de mastite e bioindicador da qualidade do leite, pesquisadores sugerem o estabelecimento de uma metodologia de quantificação desta proteína aplicável ao seu monitoramento em tanques de refrigeração em laticínios.

A nova tecnologia a ser gerada deve ser de baixo custo, realizar a quantificação rápida da proteína, assim como apresentar um valor de ponto de corte confiável para a haptoglobina, de forma a classificar amostras e lotes de leite conforme o seu padrão de qualidade (Akerstedt et al., 2009; Akerstedt et al., 2011; Tan et al., 2012).

\section{Haptoglobina no contexto do bem-estar e estresse animal}

Embora elevados níveis de haptoglobina sanguínea sejam indicativos confiáveis da ocorrência de processos infecciosos ou inflamatórios em ruminantes, pesquisadores suspeitaram que quadros de estresse também poderiam acarretar na elevação da concentração sérica desta proteína, visto que o estresse constitui uma síndrome que altera diversos parâmetros fisiológicos (Sporer et al., 2008; Ndou et al., 2011). O primeiro trabalho nesse âmbito foi publicado em 1993, no qual se avaliou o nível de estresse de bovinos transportados pelo percurso de $1.400 \mathrm{Km}$; após o desembarque dos animais, constatou-se significante elevação da haptoglobina plasmática e que se manteve alta por pelo menos 24 horas (Murata \& Miyamoto 1993). Estes resultados foram comprovados por pesquisas posteriores, sendo demonstrado que os níveis desta proteína se elevam a partir de 6 a 12 horas após o início do estimulo estressante e se mantêm altos pelo período que ele ocorre (Arthington et al., 2003; Mitchell et al., 2008; Lomborg et al., 2008).

Uma pesquisa realizada na Espanha demonstrou que o estresse gerado pelo transporte pode ser iniciado antes mesmo do embarque dos bovinos; o uso de técnicas de manejo inadequadas para agrupamento dos animais induziu uma resposta de fase aguda, com nível médio de haptoglobina sérica de $0,43 \mathrm{~g} / \mathrm{L}$. Ao final do transporte por $730 \mathrm{~km} \mathrm{em} 27$ horas, a sua concentração já se encontrava em $0,78 \mathrm{~g} / \mathrm{L}$ (Averós et al., 2008). Diante da nítida associação entre estresse e resposta de fase aguda, pesquisadores buscaram detalhar a relação entre $o$ tempo do transporte, a distância percorrida e os níveis de haptoglobina plasmática. Foram realizados transportes de bovinos por $6 \mathrm{~h}(280$ $\mathrm{km}), 9 \mathrm{~h}(435 \mathrm{~km}), 12 \mathrm{~h}(582 \mathrm{~km}), 18 \mathrm{~h}(902 \mathrm{~km})$ e $24 \mathrm{~h}(1192 \mathrm{~km})$, sendo constatado que o transporte por 18 a 24 horas eleva a haptoglobina significantemente para valores acima de $0,40 \mathrm{~g} / \mathrm{L}$ (Earley et al., 2010; Earley et al., 2013), independente se os animais são transportados por via terrestre ou marítima (Earley et al., 2012).

Estudos realizados com ovinos obtiveram resultados semelhantes a bovinos, reforçando a ligação entre os níveis de haptoglobina e o bemestar animal; animais com estresse após transporte apresentaram níveis elevados desta proteína, com valor médio de 0,56 g/L (Kostro et al., 2009), podendo as concentrações chegar a valores médios de 1,0 g/L (Tadich et al., 2009). Contudo, foi descoberto que sob influência do estresse por transporte, os níveis de haptoglobina nesta espécie permanecem elevados por até $48 \mathrm{~h}$ após o desembarque, indicando que estes animais necessitam de tempo maior que os bovinos para reestabelecer suas funções fisiológicas normais e poderem ser encaminhados para abate (Piccione et al., 2012).

Embora o estresse não seja uma condição que represente risco à segurança alimentar e saúde pública diretamente, o segmento da indústria de carnes constatou que o estresse pré-abate pode resultar em grandes prejuízos à qualidade da carne (Silva et al., 2014), envolvendo a elevação do seu pH para valores acima de 5,8, maior retenção de líquidos e coloração alterada, que acarretam em maior probabilidade de deterioração microbiana; tais produtos recebem a denominação de "Darkcutting beef" (carne bovina de corte escuro) ou "Dark, firm and dried - DFD" (escura, firme e seca) (Ferguson \& Warner 2008; Weglarz, 2010; Costa, 2012). Pesquisadores realizaram um trabalho de comparação entre os valores de haptoglobina de bovinos em condições de estresse antes do abate e seus respectivos valores de $\mathrm{pH}$ da carne durante o processo de produção; constatouse uma correlação positiva significante ( $\mathrm{p}$ menor que 0,01 ) entre estas variáveis (Campo et al., 2008).

Outras condições estressantes que induzem a elevação significante da haptoglobina plasmática são o estado de ansiedade gerado pelo período préparto em vacas (Alsemgeest et al., 1993; Huzzey 
et al., 2015) e restrições nutricionais em bezerros (Katoh et al., 2002). Estudos realizados no período de desmame indicaram que o evento da apartação não altera a concentração de haptoglobina sanguínea nos bezerros (Hickey et al., 2003); contudo, vacas separadas abruptamente de suas crias apresentaram elevação significante da haptoglobina plasmática entre 2 a 35 dias após o evento, sendo registrados valores entre $0,43 \mathrm{~g} / \mathrm{L} \mathrm{e}$ 0,72 g/L (Lynch et al., 2010).

\section{Considerações finais}

Frente às informações apresentadas, é evidente o potencial que a haptoglobina apresenta como bioindicador de saúde animal em diferentes segmentos da cadeia produtiva pecuária. $\mathrm{Na}$ pecuária de corte, a quantificação desta proteína pode contribuir com a triagem dos animais na inspeção ante morten, permitindo a identificação dos animais com doença sub-clínica que não foram diagnosticados pela inspeção visual; pode também contribuir para a verificação do bem-estar animal nos momentos após o transporte e préabate, evitando-se o abate de animais com possíveis quadros de estresse que possam alterar a qualidade dos produtos cárneos. $\mathrm{Na}$ pecuária leiteira, a haptoglobina pode contribuir diretamente com a identificação de vacas com diferentes níveis de mastite clínica e sub-clínica, além de permitir a identificação de lotes de leite com alta concentração de células somática ou oriunda de rebanhos acometidos por agentes infecciosos.

Todo este potencial de utilidade se deve justamente ao caráter inespecífico da resposta de fase aguda que induz a produção e secreção da haptoglobina nos fluidos biológicos animais, independente da causa do quadro inflamatório ou infeccioso.

Para que se torne uma ferramenta de amplo uso, tanto por médicos veterinários dos Sistemas Oficiais de Inspeção, como pelos próprios estabelecimentos industriais de produtos de origem animal, recomenda-se a realização de estudos de validação do uso da haptoglobina como bioindicador de saúde animal em parceria com empresas do setor. Assim, sua utilidade prática seja verificada diretamente pelos profissionais envolvidos.

\section{Agradecimentos}

Os autores agradecem à Fundação de Apoio à Pesquisa e à Extensão (FAPEX) e ao Conselho
Nacional de Desenvolvimento Científico e Tecnológico (CNPq).

\section{Referências Bibliográficas}

Akerstedt, M., Björck, L., Persson Waller, K. \& Sternesjö, A. 2006. Biosensor assay for determination of haptoglobin in bovine milk. The Journal of Dairy Research, 73, 299-305.

Akerstedt, M., Forsback, L., Larsen, T. \& Svennersten-Sjaunja, K. 2011. Natural variation in biomarkers indicating mastitis in healthy cows. The Journal of Dairy Research, 78, 88-96.

Akerstedt, M., Persson Waller, K. \& Sternesjö, A. 2007. Haptoglobin and serum amyloid A in relation to the somatic cell count in quarter, cow composite and bulk tank milk samples. The Journal of Dairy Research, 74, 198-203.

Akerstedt, M., Waller, K. P., Larsen, L. B., Forsbäck, L. \& Sternesjö, Å. 2008. Relationship between haptoglobin and serum amyloid A in milk and milk quality. International Dairy Journal, 18, 669-674.

Akerstedt, M., Waller, K. P. \& Sternesjö, A. 2009. Haptoglobin and serum amyloid A in bulk tank milk in relation to raw milk quality. Journal of Dairy Research, 76, 483-489.

Alsemgeest, S. P., Taverne, M. A., Boosman, R., van der Weyden, B. C. \& Gruys, E. 1993. Peripartum acute-phase protein serum amyloid-A concentration in plasma of cows and fetuses. American Journal of Veterinary Research, 54, 164-167.

Arredouani, M., Matthijs, P., Van Hoeyveld, E., Kasran, a, Baumann, H., Ceuppens, J. L. \& Stevens, E. 2003. Haptoglobin directly affects $\mathrm{T}$ cells and suppresses $\mathrm{T}$ helper cell type 2 cytokine release. Immunology, 108, 144-51.

Arredouani, M. S., Kasran, A., Vanoirbeek, J. a, Berger, F. G., Baumann, H. \& Ceuppens, J. L. 2005. Haptoglobin dampens endotoxininduced inflammatory effects both in vitro and in vivo. Immunology, 114, 263-71.

Arthington, J. D., Eicher, S. D., Kunkle, W. E. \& Martin, F. G. 2003. Effect of transportation and commingling on the acute-phase protein response, growth, and feed intake of newly weaned beef calves. Journal of Animal Science, 81, 1120-1125.

Averós, X., Martín, S., Riu, M., Serratosa, J. \& Gosálvez, L. F. 2008. Stress response of extensively reared young bulls being 
transported to growing-finishing farms under Spanish summer commercial conditions. Livestock Science, 119, 174-182.

Bandeira, F. S., Picoli, T., Zani, J. L., Silva, W. P. \& Fischer, G. 2013. Frequência de Staphylococcus aureus em casos de mastite bovina subclínica, na região sul do Rio Grande do Sul. Arquivos Do Instituto Biológico, 80, 1 6.

Battisti, M. K. B., Silva, D. M. da, Reusing, M. S. de O., Beltrame, O. C., Schmidt, E. M. S., Fagliari, J. J., Dittrich, R. L. \& Guérios, S. D. 2013. Proteínas de fase aguda em cadelas com neoplasia mamária. Ciência Rural, 43, 902 907.

Blagojevic, B., Antic, D., Ducic, M. \& Buncic, S. 2011. A study of haptoglobin levels in groups of cattle and pigs with and without abnormalities at meat inspection. Foodborne Pathogens and Disease, 8, 1119-1124.

Campo, M., Lima, J. M. S., Brito, G., Manteca, X., Hernández, P., Sañudo, C. \& Barcelona, U. A. De. 2008. Effect of different feeding strategies on animal welfare and meat quality in Uruguayan steers. In: Proceedings of the 54th International Congress of Meat Science and Technology, Cape Town, South Africa.

Carneiro, D. M. V. F., Domingues, P. F. \& Vaz, A. K. 2009. Imunidade inata da glândula mamária bovina: resposta à infecção. Ciência Rural, 39, 1934-1943.

Carvalho, V. S., Araújo, B. R., Vasconcelos, T. C., Chiminazzo, C., Neto, A. O. C., Ayres, M. C. C., Guimarães, J. E. \& Costa, J. N. 2012. Evolução clínica e avaliação de parâmetros leucocitários e de proteínas de fase aguda na pododermatite infecciosa ovina. Pesquisa Veterinária Brasileira, 32, 1289-1296.

Ceciliani, F., Ceron, J. J., Eckersall, P. D. \& Sauerwein, H. 2012. Acute phase proteins in ruminants. Journal of Proteomics, 75, 42074231.

Cooke, R. F. \& Arthington, J. D. 2012. Concentrations of haptoglobin in bovine plasma determined by ELISA or a colorimetric method based on peroxidase activity. Journal of Animal Physiology and Animal Nutrition, 97, 531-536.

Costa, R. S. 2012. Characterization of rigor mortis of longissimus dorsi and triceps brachii muscles of male cattle carcasses. African Journal of Biotechnology, 11, 8127-8132.
Czopowicz, M., Szaluś-Jordanow, O., Mickiewicz, M., Moroz, A., Witkowski, L., Markowska-Daniel, I., Reczyńska, D., Bagnicka, E. \& Kaba, J. 2017. Agreement between commercial assays for haptoglobin and serum amyloid A in goats. Acta Veterinaria Scandinavica, 59:65.

Earley, B., Drennan, M. \& O’Riordan, E. G. 2013. The effect of road transport in comparison to a novel environment on the physiological, metabolic and behavioural responses of bulls. Research in Veterinary Science, 95, 811-818.

Earley, B., Murray, M. \& Prendiville, D. J. 2010. Effect of road transport for up to 24 hours followed by twenty-four hour recovery on live weight and physiological responses of bulls. BMC Veterinary Research, 6, 38.

Earley, B., Murray, M., Prendiville, D. J., Pintado, B., Borque, C. \& Canali, E. 2012. The effect of transport by road and sea on physiology, immunity and behaviour of beef cattle. Research in Veterinary Science, 92, 531-541.

Eaton, J. W., Brandt, P., Mahoney, J. R. \& Lee, J. T. J. 1982. Haptoglobin: a natural bacteriostat. Science, 215, 691-693.

Eckersall, P. D., Duthie, S., Toussaint, M. J. M., Gruys, E., Heegaard, P., Alava, M., Lipperheide, C. \& Madec, F. 1999. Standardization of diagnostic assays for animal acute phase proteins. Advances in Veterinary Medicine, 41, 643-655.

Eckersall, P. D., Lawson, F. P., Bence, L., Waterston, M. M., Lang, T. L., Donachie, W. \& Fontaine, M. C. 2007. Acute phase protein response in an experimental model of ovine caseous lymphadenitis. BMC Veterinary Research, 3, 1-6.

Eckersall, P. D., Young, F. J., McComb, C., Hogarth, C. J., Safi, S., Weber, A., McDonald, T., Nolan, A. M. \& Fitzpatrick, J. L. 2001. Acute phase proteins in serum and milk from dairy cows with clinical mastitis. Veterinary Record, 148, 35-41.

Eckersall, P. D., Young, F. J., Nolan, A. M., Knight, C. H., McComb, C., Waterston, M. M., Hogarth, C. J., Scott, E. M. \& Fitzpatrick, J. L. 2006. Acute phase proteins in bovine milk in na experimental model of Staphylococcus aureus subclinical mastitis. Journal of Dairy Science, 89, 1488-1501.

Elson, E. C. 1974. Quantitative determination of serum haptoglobin. A simple and rapid method. American Journal of Clinical 
Pathology, 62, 655-663.

Ferguson, D. M. \& Warner, R. D. 2008. Have we underestimated the impact of pre-slaughter stress on meat quality in ruminants? Meat Science, 80, 12-19.

Filippo, P. A. Di, Gomes, F. R., Mascarenhas, L. S., Almeida, A. J. de, \& Rodrigues, A. B. F. 2014. Proteinograma sérico e do líquido peritoneal de equinos submetidos à orquiectomia. Ciência Rural, 44, 2221-2227.

Gómez-Laguna, J., Salguero, F. J., Pallarés, F. J., Rodríguez-gómez, I. M., Barranco, I. \& Carrasco, L. 2011. Acute phase proteins as biomarkers in animal health and welfare. In: Veas, F. (ed.) Acute Phase Proteins as Early Non-Specific Biomarkers of Human and Veterinary Diseases. InTech, DOI: 10.5772/19166.

Grönlund, U., Hultén, C., Eckersall, P. D., Hogarth, C. \& Persson Waller, K. 2003. Haptoglobin and serum amyloid A in milk and serum during acute and chronic experimentally induced Staphylococcus aureus mastitis. Journal of Dairy Research, 70, 379-386.

Grönlund, U., Sandgren, C. H. \& Waller, K. P. 2005. Haptoglobin and serum amyloid A in milk from dairy cows with chronic sub-clinical mastitis. Veterinary Research, 36, 191-198.

Gruys, E., Toussaint, M. J. M., Niewold, T. A, \& Koopmans, S. J. 2005. Acute phase reaction and acute phase proteins. Journal of Zhejiang University. Science B, 6, 1045-56.

Hickey, M. C., Drennan, M. \& Earley, B. 2003. The effect of abrupt weaning of suckler calves on the plasma concentrations of cortisol, catecholamines, leukocytes, acute-phase proteins and in vitro interferon-gamma production. Journal of Animal Science, 81, 2847-55.

Hill, A. A., Horigan, V., Clarke, K. a., Dewé, T. C. M., Stärk, K. D. C., O'Brien, S. \& Buncic, S. 2014. A qualitative risk assessment for visual-only post-mortem meat inspection of cattle, sheep, goats and farmed/wild deer. Food Control, 38, 96-103.

Hirvonen, J. 1997. Acute phase response in emergency slaughtered dairy cows. Meat Science, 46, 249-257.

Hirvonen, J., Eklund, K., Teppo, A. M., Huszenicza, G., Kulcsar, M., Saloniemi, H. \& Pyorala, S. 1999. Acute phase response in dairy cows with experimentally induced Escherichia coli mastitis. Acta Veterinaria Scandinavica, 40, 35-46.

Hisaeda, K., Arima, H., Sonobe, T., Nasu, M., Hagiwara, K., Kirisawa, R., Takahashi, T., Kikuchi, N. \& Nagahata, H. 2011. Changes in acute-phase proteins and cytokines in serum and milk whey from dairy cows with naturally occurring peracute mastitis caused by Klebsiella pneumoniae and the relationship to clinical outcome. Journal of Veterinary Medical Science, 73, 1399-1404.

Hiss, S., Mielenz, M., Bruckmaier, R. M. \& Sauerwein, H. 2004. Haptoglobin concentrations in blood and milk after endotoxin challenge and quantification of mammary Hp mRNA expression. Journal of Dairy Science, 87, 3778-3784.

Höfner, M. C., Fosbery, M. W., Eckersall, P. D. \& Donaldson, A. I. 1994. Haptoglobin response of cattle infected with foot-and-mouth disease virus. Research in Veterinary Science, 57, 125-128.

Horadagoda, N. U., Knox, K. M., Gibbs, H. a, Reid, S. W., Horadagoda, A., Edwards, S. E. \& Eckersall, P. D. 1999. Acute phase proteins in cattle: discrimination between acute and chronic inflammation. Veterinary Record, 144, 437-441.

Huzzey, J. M., Mann, S., Nydam, D. V., Grant, R. J. \& Overton, T. R. 2015. Associations of peripartum markers of stress and inflammation with milk yield and reproductive performance in Holstein dairy cows. Preventive Veterinary Medicine, 120, 291-297.

Kalmus, P., Simojoki, H., Pyörälä, S., Taponen, S., Holopainen, J. \& Orro, T. 2013. Milk haptoglobin, milk amyloid $\mathrm{A}$, and $\mathrm{N}$-acetyl- $\beta$ d-glucosaminidase activity in bovines with naturally occurring clinical mastitis diagnosed with a quantitative PCR test. Journal of Dairy Science, 96, 3662-3670.

Katoh, N., Oikawa, S., Oohashi, T., Takahashi, Y. \& Itoh, F. 2002. Decreases of apolipoprotein B-100 and A-I concentrations and induction of haptoglobin and serum amyloid A in nonfed calves. Journal of Veterinary Medical Science, 64, 51-55.

Klauke, T. N., Gronewold, T. M. A., Perpeet, M., Plattes, S. \& Petersen, B. 2013. Measurement of porcine haptoglobin in meat juice using surface acoustic wave biosensor technology. Meat Science, 95, 699-703.

Kostro, K., Jarosz, L., Gruszecki, T., Junkuszew, 
A. \& Lipecka, C. 2009. Utility of haptoglobin assay for sheep welfare and health status evaluation in pre- and postslaughter period. Bulletin of the Veterinary Institute in Pulawy, 53, 111-116.

Kurash, J. K., Shen, C.-N. \& Tosh, D. 2004. Induction and regulation of acute phase proteins in transdifferentiated hepatocytes. Experimental Cell Research, 292, 342-358.

Lai, I. H., Tsao, J. H., Lu, Y. P., Lee, J. W., Zhao, X., Chien, F. L. \& Mao, S. J. T. 2009. Neutrophils as one of the major haptoglobin sources in mastitis affected milk. Veterinary Research, 40, 17.

Langoni, H. 2013. Qualidade do leite: Utopia sem um programa sério de monitoramento da ocorrência de mastite bovina. Pesquisa Veterinaria Brasileira, 33, 620-626.

Laurell, C. B. \& Nyman, M. 1957. Studies on the serum haptoglobin level in hemoglobinemia and its influence on renal excretion of hemoglobin. Blood, 12, 493-506.

Lomborg, S. R., Nielsen, L. R., Heegaard, P. M. H. \& Jacobsen, S. 2008. Acute phase proteins in cattle after exposure to complex stress. Veterinary Research Communications, 32, $575-82$.

Lynch, E. M., Earley, B., McGee, M. \& Doyle, S. 2010. Characterisation of physiological and immunological responses in beef cows to abrupt weaning and subsequent housing. $B M C$ Veterinary Research, 6, 37.

Meling, S., Bardsen, K. \& Ulvund, M. J. 2012. Presence of an acute phase response in sheep with clinical classical scrapie. BMC Veterinary Research, 8, 1 .

Middleton, J. R., Hardin, D., Steevens, B., Randle, R. \& Tyler, J. W. 2004. Use of somatic cell counts and California mastitis test results from individual quarter milk samples to detect subclinical intramammary infection in dairy cattle from a herd with a high bulk tank somatic cell count. Journal of the American Veterinary Medical Association, 224, 419-423.

Mitchell, G. B., Clark, M. E., Siwicky, M. \& Caswell, J. L. 2008. Stress alters the cellular and proteomic compartments of bovine bronchoalveolar lavage fluid. Veterinary Immunology and Immunopathology, 125, 111125.

Murata, H. \& Miyamoto, T. 1993. Bovine haptoglobin as a possible immunomodulator in the sera of transported calves. British Veterinary Journal, 149, 277-283.

Murata, H., Shimada, N. \& Yoshioka, M. 2004. Current research on acute phase proteins in veterinary diagnosis: An overview. Veterinary Journal, 168, 28-40.

Ndou, S. P., Muchenje, V. \& Chimonyo, M. 2011. Animal welfare in multipurpose cattle production systems and its implications on beef quality. African Journal of Biotechnology, 10, 1049-1064.

Nielsen, B. H., Jacobsen, S., Andersen, P. H., Niewold, T. A. \& Heegaard, P. M. H. 2004. Acute phase protein concentrations in serum and milk from healthy cows, cows with clinical mastitis and cows with extramammary inflammatory conditions. Veterinary Record, 154, 361-365.

Ohtsuka, H., Kudo, K., Mori, K., Nagai, F., Hatsugaya, A., Tajima, M., Tamura, K., Hoshi, F., Koiwa, M. \& Kawamura, S. 2001. Acute phase response in naturally occurring coliform mastitis. Journal of Veterinary Medical Science, 63, 675-678.

Petersen, H. H., Nielsen, J. P. \& Heegaard, P. M. H. 2004. Application of acute phase protein measurements in veterinary clinical chemistry. Veterinary Research, 35, 163-187.

Piccione, G., Casella, S., Giannetto, C., Giudice, E. \& Fazio, F. 2012. Utility of acute phase proteins as biomarkers of transport stress in ewes. Small Ruminant Research, 107, 167171.

Pyörälä, S., Hovinen, M., Simojoki, H., Fitzpatrick, J., Eckersall, P. D. \& Orro, T. 2011. Acute phase proteins in milk in naturally acquired bovine mastitis caused by different pathogens. Veterinary Record, 168, 535.

Rossbacher, J., Wagner, L. \& Pasternack, M. S. 1999. Inhibitory effect of haptoglobin on granulocyte chemotaxis, phagocytosis and bactericidal activity. Scandinavian Journal of Immunology, 50, 399-404.

Safi, S., Khoshvaghti, A., Jafarzadeh, S. R., Bolourchi, M. \& Nowrouzian, I. 2009. Acute phase proteins in the diagnosis of bovine subclinical mastitis. Veterinary Clinical Pathology, 38, 471-476.

Saini, P. K., Riaz, M., Webert, D. W., Eckersall, P. D., Young, C. R., Stanker, L. H., Chakrabarti, E. \& Judkins, J. C. 1998. Development of a simple enzyme 
immunoassay for blood haptoglobin concentration in cattle and its application in improving food safety. American Journal of Veterinary Research, 59, 1101-1107.

Saini, P. K. \& Webert, D. W. 1991. Application of acute phase reactants during antemortem and postmortem meat inspection. Journal of the American Veterinary Medical Association, 198, 1898-1901.

Salonen, M., Hirvonen, J., Pyörälä, S., Sankari, S. \& Sandholm, M. 1996. Quantitative determination of bovine serum haptoglobin in experimentally induced Escherichia coli mastitis. Research in Veterinary Science, 60, 88-91.

Sargeant, J. M., Leslie, K. E., Shirley, J. E., Pulkrabek, B. J. \& Lim, G. H. 2001. Sensitivity and specificity of somatic cell count and California Mastitis Test for identifying intramammary infection in early lactation. Journal of Dairy Science, 84, 2018-2024.

Segawa, T., Amatsuji, H., Suzuki, K., Suzuki, M., Yanagisawa, M., Itou, T., Sakai, T. \& Nakanishi, T. 2013. Molecular characterization and validation of commercially available methods for haptoglobin measurement in bottlenose dolphin. Results in Immunology, 3, $57-63$.

Sharifiyazdia, H., Nazifi, S., Nikseresht, K. \& Shahriari, R. 2012. Evaluation of Serum Amyloid A and Haptoglobin in Dairy Cows Naturally Infected with Brucellosis. Journal of Bacteriology \& Parasitology, 3, 157.

Silva, B., Poleti, M. D., Moncau, C. T., Rosa, A. F., Silva, S. D. L. \& Baliero, J. C. D. C. 2014. Características endócrinas, metabólicas e indicadoras da qualidade da carne em bovinos Nelore castrados e não castrados. Ciência Rural, 44, 904-910.

Singh, M., Sharma, A., Sharma, R., Mittal, D., Yadav, P. \& Charaya, G. 2015. Estimation of acute phase proteins as early biomarkers of buffalo subclinical mastitis. Asian Journal of Animal and Veterinary Advances, 10, 894902.

Skinner, J. G., Brown, R. A. \& Roberts, L. 1991. Bovine haptoglobin response in clinically defined field conditions. Veterinary Record, 128, 147-149.

Soler, L., Gutiérrez, a., Müllebner, a., Cerón, J. J. \& Duvigneau, J. C. 2013. Towards a better understanding of salivary and meat juice acute phase proteins determination in pigs: An expression study. Veterinary Immunology and Immunopathology, 156, 91-98.

Sporer, K. R. B., Weber, P. S. D., Burton, J. L., Earley, B. \& Crowe, M. A. 2008. Transportation of young beef bulls alters circulating physiological parameters that may be effective biomarkers of stress. Journal of Animal Science, 86, 1325-1334.

Stenfeldt, C., Heegaard, P. M., Stockmarr, A., Tjørnehøj, K. \& Belsham, G. J. 2011. Analysis of the acute phase responses of Serum Amyloid A, Haptoglobin and Type 1 Interferon in cattle experimentally infected with foot-and-mouth disease virus serotype O. Veterinary Research, 42,66 .

Suojala, L., Orro, T., Järvinen, H., Saatsi, J. \& Pyörälä, S. 2008. Acute phase response in two consecutive experimentally induced $E$. coli intramammary infections in dairy cows. Acta Veterinaria Scandinavica, 50, 18.

Tadich, N., Gallo, C., Brito, M. L. \& Broom, D. M. 2009. Effects of weaning and $48 \mathrm{~h}$ transport by road and ferry on some blood indicators of welfare in lambs. Livestock Science, 121, 132136.

Tan, X., Ding, S.-Q., Hu, Y.-X., Li, J.-J. \& Zhou, J.-Y. 2012. Development of an immunosensor assay for detection of haptoglobin in mastitic milk. Veterinary Clinical Pathology, 41, 575581.

Theilgaard-Mönch, K., Jacobsen, L. C., Nielsen, M. J., Rasmussen, T., Udby, L., Gharib, M., Arkwright, P.D., Gombart, A.F., Calafat, J., Moestrup, S.K., Porse, B.T. \& Borregaard, N. 2006. Haptoglobin is synthesized during granulocyte differentiation, stored in specific granules, and released by neutrophils in response to activation. Blood, 108, 353-361.

Thielen, M. A., Mielenz, M., Hiss, S. \& Sauerwein, H. 2005. Qualitative detection of haptoglobin mRNA in bovine and human blood leukocytes and bovine milk somatic cells. Veterinární Medicína, 50, 515-520.

Thielen, M. A., Mielenz, M., Hiss, S., Zerbe, H., Petzl, W., Schuberth, H. J., Seyfert, H. M. \& Sauerwein, H. 2007. Short communication: Cellular localization of haptoglobin mRNA in the experimentally infected bovine mammary gland. Journal of Dairy Science, 90, 12151219.

Tothova, C., Nagy, O. \& Kovac, G. 2014. Acute phase proteins and their use in the diagnosis of diseases in ruminants : a review. Veterinární 
Medicina, 59, 163-180.

Tourlomoussis, P., Eckersall, P. D., Waterson, M. M. \& Buncic, S. 2004. A comparison of acute phase protein measurements and meat inspection findings in cattle. Foodborne Pathogens and Disease, 1, 281-290.

Vels, L., Rontved, C. M., Bjerring, M. \& Ingvartsen, K. L. 2009. Cytokine and acute phase protein gene expression in repeated liver biopsies of dairy cows with a lipopolysaccharide-induced mastitis. Journal of Dairy Science, 92, 922-934.

Weglarz, A. 2010. Meat quality defined based on $\mathrm{pH}$ and colour depending on cattle category and slaughter season. Czech Journal of Animal Science, 55, 548-556.
Wenz, J. R., Fox, L. K., Muller, F. J., Rinaldi, M., Zeng, R. \& Bannerman, D. D. 2010. Factors associated with concentrations of select cytokine and acute phase proteins in dairy cows with naturally occurring clinical mastitis. Journal of Dairy Science, 93, 2458-2470.

\section{Article History:}

Received 11 November 2017

Accepted 4 January 2018

Available online 12 February 2018

License information: This is an open-access article distributed under the terms of the Creative Commons Attribution License 4.0, which permits unrestricted use, distribution, and reproduction in any medium, provided the original work is properly cited. 